\title{
The discovery of correlation observed among the cell elasticity and endocrine elasticity in breast cancer constitutes a revolution for knowledge and treatment of cancer
}

\begin{abstract}
Introduction: For some decades now, studies of cell biology represent the main objective in cancer investigation for the development of new therapies, but the endocrine environment of the patient has not been taken into account because nothing has been discovered about it so far. In this paper we analyze the correlation between the degree of differentiation of the breast cancer tumor cells and metastatic lymph node and the endocrine elasticity of cholesterols in breast cancer expressed by the waste of cholesterol formula that was recently published in the journal Cancer Prevention and Current Research.
\end{abstract}

Material and methods: 375 clinical records from the III Congress hospital of patients affected by breast cancer were reviewed from 2001 to 2006. Biopsies were performed in the Leon Cuervo Rubio Hospital and in the Abel Santamaria Hospital. After the operation and before starting chemotherapy treatment patients were submitted to blood samples for study of cholesterols. The formula of the waste of cholesterol was applied. Patients were classified as a positive waste of cholesterol or negative waste of cholesterol and the result of the formula was expressed in how many times the cholesterol waste for estrogens formation was increased or decreased. The tumors were classified as well, moderately or poorly differentiated. That way any histology was classified as well differentiated, positive or negative; moderately differentiated, positive or negative; and poorly differentiated, positive or negative. The presence or not of metastatic lymph node was also analyzed.

Results: It could be seen that when the tumor is well differentiated the waste of cholesterol is around zero, which represents the endocrine metabolic balance. On the other hand, the moderately differentiated tumor cells are in outermost equilibrium and when cells are undifferentiated are even in more outermost equilibrium. This situation happens in the negative waste of cholesterol and in the positive waste of cholesterol, which shows us that in breast cancer there are a misbalance of the endocrine metabolic system, and when we are far from the equilibrium cells tend to be less differentiated; that represents a jump in breast cancer treatment where we have to keep the balance of the endocrine metabolic system for the cure. On the other hand, no matter the differentiation degree, the presence of axillary metastatic lymph node increases the misbalance. The waste of cholesterol was compared with the estrogens receptor status what means that immune histochemical have a coincidence with the waste of cholesterol positive or negative. All these findings open a new era for breast cancer treatment.
Volume 9 Issue 3 - 2018

\author{
Lazo R,' López S,' Martínez W, 'Aguiar \\ Y,' Vigil V, ${ }^{3}$ Torres F, Pileta $A,{ }^{4}$ Cabrera \\ $\mathrm{G},{ }^{4}$ Rodríguez LRodríguez M, ${ }^{3}$ Musa M, ${ }^{4}$ \\ Hernández,,3 Lazo A, ${ }^{,}$Martínez Miche ${ }^{1,5}$ Lazo \\ $M,{ }^{5}$ Martinez Jadier ${ }^{6}$ \\ 'Unidad Oncológica de Pinar del Río, Cuba \\ ${ }^{2}$ Hospital León Cuervo Rubio, Cuba \\ ${ }^{3}$ Hospital III Congreso, Cuba \\ ${ }^{4}$ Hospital Abel Santamaría, Cuba \\ ${ }^{5}$ Facultad de ciencias médicas de Pinar del Rio, Cuba \\ ${ }^{6}$ Independent technician in information technology, Cuba \\ ${ }^{7}$ Instituto Nacional de Oncología, Cuba
}

Correspondence: Roberto Lucio Lazo Carreras, Unidad Oncológica de Pinar del Río, Cuba,

Email carreraslazzo@gmail.com

Received: April 02, 2018| Published: June 06, 2018

\section{Introduction}

In 2001 we presented at the St Gallen conference Adjuvant Therapy of Primary Breast Cancer the formula of the waste of cholesterol. In that formula the values of the total cholesterol and HDL cholesterol of the patient are introduced with a computer program after operation and before starting systemic treatment. That way patients are classified as waste of cholesterol positive or negative according to the levels of total cholesterol and Hdl cholesterol according to the age, and how many times the cholesterol waste is decreased or increased. The normal values of the waste of cholesterol for estrogens formation were examined in the relation between seric cholesterol and estrogens compared to estrogen receptors in the patients by the charcoal dextran method. Afterwards it was compared with the normal levels of cholesterol in women reported in the literature according to the age, so that we can consider in the elderly the physiological increase of LDL cholesterol. That way we tried to know the balance of the human body, in this case the women taking into account the physiology of the women according to age as menarche, menopause and elderly. The results of survival in breast cancer were presented in St Gallen in patients without adjuvant chemotherapy; ${ }^{1}$ later the results in patients that received adjuvant therapy were published in the journal Cancer Prevention and Current Research. ${ }^{2,3}$ In this paper we study the connection of the waste of cholesterol with the degree of differentiation and the presence of lymph node metastasis.

This study, as prospective and observational, started in the year 2001. Patients were selected with operable breast cancer classified and staged in a multidisciplinary medical room where oncologist, surgeons and pathologist were present. After operation and before starting adjuvant treatment total cholesterol and hdl cholesterol was indicated along with the other blood test. Then the formula of the waste of cholesterol was applied (Figure 1) (Table 1). 
Table I Shows the different parameters for the calculation of the waste of cholesterol (CFBA). Correction factor by age (CWBA) cholesterol waste by age

\begin{tabular}{|c|c|c|c|c|c|c|c|c|}
\hline Age & CFBA & CWBA & Age & CFBA & CWBA & Age & CFBA & CWBA \\
\hline 17 & 3 & 0,3 & 39 & 8,7 & 0,51 & 61 & 0,96 & 0,054 \\
\hline 18 & 3 & 0,33 & 40 & 7,9 & 0,46 & 62 & 0,92 & 0,052 \\
\hline 19 & 4 & 0,37 & 41 & 7,2 & 0,42 & 63 & 0,91 & 0,051 \\
\hline 20 & 4,5 & 0,41 & 42 & 6,5 & 0,38 & 64 & 0,85 & 0,048 \\
\hline 21 & 5,5 & 0,44 & 43 & 5,8 & 0,34 & 65 & 0,83 & 0,047 \\
\hline 22 & 6 & 0,48 & 44 & 5,1 & 0,29 & 66 & 0,8 & 0,045 \\
\hline 23 & 6,6 & 0,53 & 45 & 4,4 & 0,26 & 67 & 0,82 & 0,046 \\
\hline 24 & 8 & 0,56 & 46 & 3,8 & 0,22 & 68 & 0,82 & 0,046 \\
\hline 25 & 8,7 & 0,61 & 47 & 3,1 & 0,18 & 69 & 0,83 & 0,047 \\
\hline 26 & 9,5 & 0,67 & 48 & 2,8 & 0,16 & 70 & 0,83 & 0,047 \\
\hline 27 & 10,2 & 0,62 & 49 & 2,2 & 0,13 & 71 & 0,72 & 0,04 \\
\hline 28 & 12,8 & 0,77 & 50 & I,8 & 0,1 & 72 & 0,7 & 0,039 \\
\hline 29 & 13,5 & 0,81 & 51 & 1,3 & 0,078 & 73 & 0,67 & 0,037 \\
\hline 30 & 9,6 & 0,87 & 52 & 1 & 0,006 & 74 & 0,65 & 0,036 \\
\hline 31 & 13,7 & 0,86 & 53 & 1 & 0,006 & 75 & 0,63 & 0,035 \\
\hline 32 & 12 & 0,84 & 54 & 1 & 0,006 & 76 & 0,61 & 0,034 \\
\hline 33 & 11 & 0,83 & 55 & 1 & 0,006 & 77 & 0,6 & 0,033 \\
\hline 34 & 10,5 & 0,74 & 56 & 1 & 0,006 & 78 & 0,52 & 0,029 \\
\hline 35 & 10,4 & 0,68 & 57 & 1 & 0,058 & 79 & 0,48 & 0,026 \\
\hline 36 & 10,3 & 0,72 & 58 & 1 & 0,057 & 80 & 0,46 & 0,025 \\
\hline 37 & 9,8 & 0,69 & 50 & 1 & 0,056 & 81 & 0,4 & 0,022 \\
\hline 38 & 9,3 & 0,54 & 60 & I & 0,056 & 82 & 0,35 & 0,019 \\
\hline
\end{tabular}

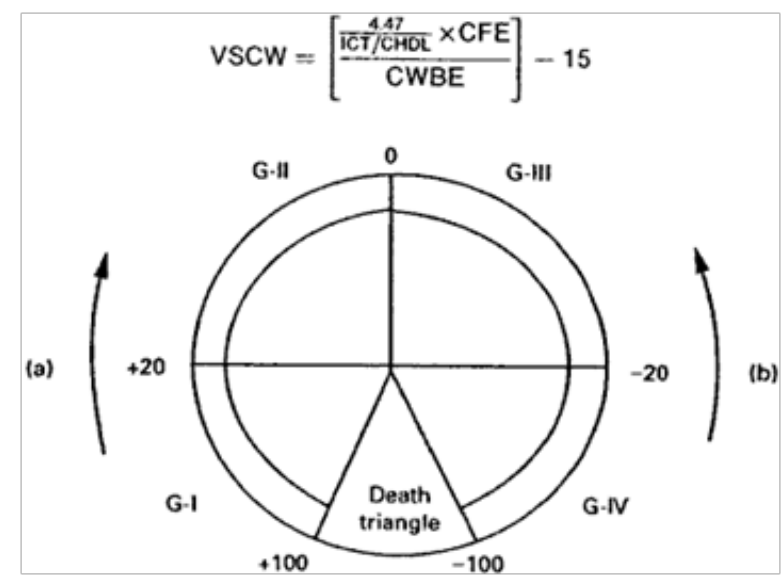

Figure I Shows the formula of the waste of cholesterol and the movement of the equilibrium (represented by zero). Results are expressed in how many times the waste of cholesterol is increased or decreased.The movement from positive to zero is performed for cytotoxic drugs, progesterone, corticoids, androgens etc. The movement from negative to zero is performed by tamoxifen, dietary with unsaturated fats, simvastatin, omega 3 etc. The death triangle represents the levels of HDL cholesterol and LDL cholesterol that are incompatible with the cell life.

\section{Material and methods}

375 clinical records of the III Congress hospital of patients affected by breast cancer were reviewed from 2001 to 2011. Biopsies were performed in the Leon Cuervo Rubio Hospital and in the Abel Santamaria Hospital. After operation and before starting chemotherapy treatment patients were submitted to blood samples for the study of cholesterols. The formula of the waste of cholesterol was applied. Patients were classified as a positive waste of cholesterol or negative waste of cholesterol and the result of the formula was expressed in how many times the cholesterol waste for estrogens formation was increased or decreased (although estrogens levels, estrogens receptors and cholesterol are not seen in the formula of the waste of cholesterol the mathematics relation is hidden in this formula and correlated with correction factor by age and with the same index 4,47). The tumors were classified as well, moderately or poorly differentiated. That way any histology was classified as well differentiated, positive or negative; moderately differentiated, positive or negative; and poorly differentiated, positive or negative. The presence or not of metastatic lymph node was also analyzed. 


\section{Results}

In this study 375 patients were evaluated. 100 patients were classified as well differentiated tumors with positive waste of cholesterol, 57patients were well differentiated with negative waste of cholesterol, 65 patients moderately differentiated with positive waste of cholesterol, 84 patients moderately with negative waste of cholesterol, 35 patients poorly differentiated with negative waste of cholesterol, and 32 poorly differentiated with positive waste of cholesterol.

It could be seen that when the tumor is well differentiated the average waste of cholesterol is around zero, which represents the endocrine metabolic balance. On the other hand, the moderately differentiated tumor cells are in outermost equilibrium and when cells are undifferentiated are even more in outermost equilibrium .This situation happens in the negative waste of cholesterol and in the positive waste of cholesterol, which shows us that the cell differentiation can be with positive waste or negative waste of cholesterol; it cannot be differentiated by the pathologist with the simple test used, but IHC test could be involved in the diagnosis as we will see later. We can see that the criteria of good and worse prognosis are maintained in the patients with negative waste of cholesterol, so the patients with negative waste of cholesterol have the more negative waste when cells are more undifferentiated, and when we see table number two that compare patients with lymph node metastasis or not, we can see clearly that in n0p (without lymph node metastasis in the axilla) the average waste is nearer zero than when patient has lymph node metastasis. It is observed in the two polar groups, well and poorly differentiated. On the other hand, in patients with positive waste of cholesterol the same happened, but inversely: patients nearer zero have well differentiated tumors, and when the tumor is more undifferentiated the waste is far from zero, but here when we analyze the lymph node metastasis the fact of having a high waste of cholesterol represents a sign of bad prognosis according to the degree of differentiation, but this misbalance is major when the patients have lymph node metastasis.

When we reviewed the levels of the waste of cholesterol we encountered 3 findings connected to immune histochemical tests. 1) $73 \%$ of the triple negative and her $2+++$ were observed in patients with positive waste of cholesterol. 2) $75 \%$ of the tumors RE+ ranged from +3 times to -10 times the normal waste of cholesterol.

Afterwards we analyzed the polar groups that were well differentiated tumors and the poorly differentiated tumors in relation to the waste of cholesterol and the presence or not of lymph node metastasis in the axillary clearance (Figures 2-4).

Table 2 shows the connection between the degree of differentiation and the waste of cholesterol (WD-well differentiated with negative waste of cholesterol; WD+, well differentiated with positive waste of cholesterol; MD, moderately with negative waste of cholesterol, moderately with positive waste of cholesterol; PD, poorly differentiated with negative waste of cholesterol; PD+, poorly differentiated with positive waste of cholesterol)

\begin{tabular}{lll}
\hline DD and Waste of cholesterol & media of the waste & n of patients \\
\hline WD- & $-3,2$ & 57 \\
WD+ & 5,6 & 100 \\
MD- & $-4,2$ & 84 \\
MD+ & 6,3 & 67 \\
PD- & -3 & 35 \\
PD+ & 7,6 & 32
\end{tabular}

Table 3 Shows the media of the waste of cholesterol according with the degree of differentiation in patients with well differentiated and poorly differentiated with positive or negative waste of cholesterol in patients with lymph node metastasis (NIbp) and in patients without lymph node metastasis (N0p)

\begin{tabular}{lllll}
\hline waste and DD & $\begin{array}{l}\text { media of waste in } \\
\mathbf{n} \mathbf{0} \mathbf{p}+\mathbf{n} \mathbf{l} \mathbf{b} \mathbf{p}\end{array}$ & $\mathbf{m}$ of waste in $\mathbf{n} \mathbf{p}$ & $\mathbf{m}$ of waste in $\mathbf{n} \mathbf{b p}$ & Total of patients \\
\hline WD- & $-3,2$ & -3 & $-3,74$ & 57 \\
WD+ & 5,6 & 5,2 & 6,61 & 35 \\
PD- & -3 & $-2,47$ & $-3,68$ & 32 \\
PD+ & 7,6 & 7,1 & 8,2
\end{tabular}




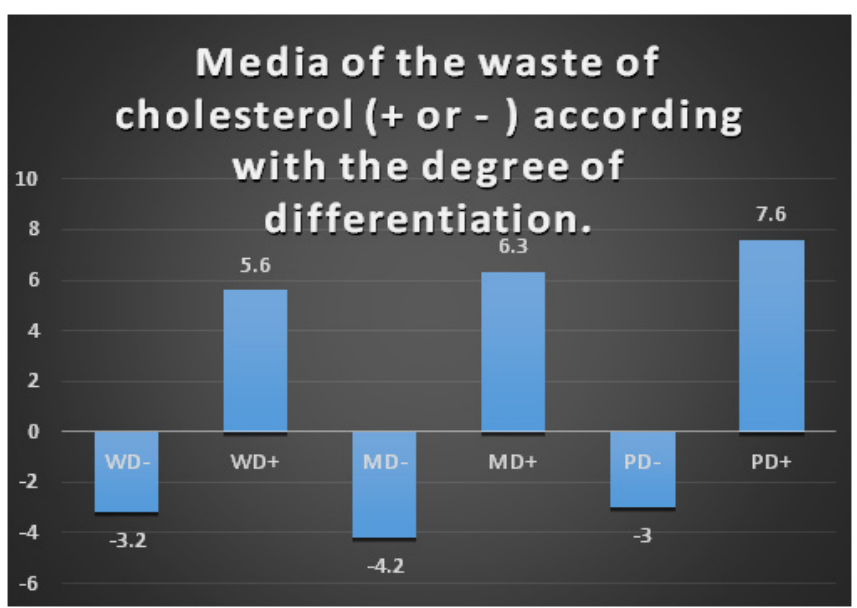

Figure 2 Shows the media of the waste of cholesterol in the different grade of differentiation WD (Well differentiated positive or negative), MD, Moderately differentiated positive or negative; PD, Poorly differentiated positive or negative.

Media of the waste of cholesterol with
the presence (N1bp ) or not (NOp ) of
axillary lymph node disection in tumors
well differentiated and poorly
differentiated.
wD+
media of waste in n0p $+\mathrm{n} 1 \mathrm{bp}$
m of waste in $n 0 p$
mo waste in $n 1 b p$

Figure 3 Shows the comparison of the levels of the waste of cholestero in the two polar groups (Well differentiated and Poorly differentiated with positive or negative waste of cholesterol) in relation with the presence of lymph node metastasis (NIbp) or not (NOp). It is expressed the levels of the waste of cholesterol without to keep in mind the lymph node metastasis $(\mathrm{NIbp}+\mathrm{N} 0 \mathrm{p})$.

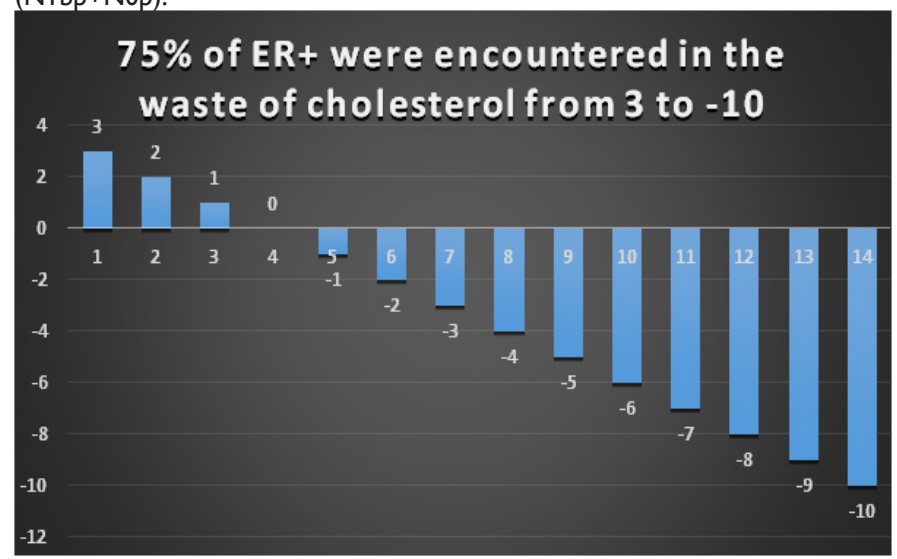

Figure 4 It can be seen that $75 \%$ of all the patients Estrogen Receptors positive are included in the waste of cholesterol from +3 times the normal waste of cholesterol until - 10 times the normal waste of cholesterol.

\section{Discussion}

\section{Results analysis}

As we see in the results, the factors of poor prognosis in breast cancer such as cell undifferentiation and the presence of metastasic lymph nodes are directly related to the levels of cholesterol waste, that is, the more differentiated the tumor, the closer it is to the equilibrium (represented by the zero in the formula), and the more undifferentiated it is the farther the equilibrium is found in both the positive and negative waste; on the other hand we find another independent factor that moves away from the normal cholesterol expenditure, which is the presence or not of axillary metastasic lymph nodes, which moves us away from equilibrium as we observe in the tables presented independently of the degree of cellular differentiation in well-differentiated and poorly differentiated tumors in both positive and negative expenses. These two elements are essential factors in the prognosis of breast cancer, intervening in the current TNM classification and therefore in current therapeutic decisions.

It is good to point out that the most important factor that measures cholesterol expenditure is the relationship between total cholesterol and HDL cholesterol, which is included in the formula for spending at the beginning of this article, that is, the levels of HDL cholesterol levels are increased in high-expenditure tumors and are decreased in low-expenditure tumors.

What does it mean that the Hdl is increased or decreased? First, the cholesterol expenditure formula was made taking into account the normal values of total cholesterol and normal HDL cholesterol according to age reported in the medical literature in women. Previous studies conducted by us found a direct relationship between hdl cholesterol and estrogen and estrogen receptors measured by the carbon-dextran method in patients with breast cancer, thus emerging the concept of cholesterol expenditure for the formation of estrogens and it was found that the waste of cholesterol for the formation of estrogens was inversely proportional to total cholesterol levels, of mode than patients with high cholesterol expenditure in the process of steroidogenesis have low serum levels of total cholesterol and increased HDL cholesterol; the opposite happened in low-waste tumors where high levels of total cholesterol were found, low hdl, low levels of serum estrogens and estrogen receptors were empty, so they were measurable and therefore were reported as positive estrogen receptors. This evidenced the role of HDL cholesterol in the formation of estrogens in the process of steroidogenesis ${ }^{4}$ where there is a direct relationship between them, which makes us suppose that the HDL controls the formation of estrogens in the steroidogenesis either because it forms it directly or because its levels by an unknown mechanism make it possible for LDL cholesterol to intervene in the steroid genesis for the formation of estrogen. If we analyze this aspect, we see that from the theoretical point of view there is a breast cancer with high levels of HDL cholesterol and another with low levels of HDL cholesterol. If we see previous studies like the one presented in St Gallen in 2001, we see how high-expenditure tumors which have high levels of HDL cholesterol are the most aggressive ${ }^{1}$ since they metastasize faster and that agrees with the studies carried out by Chritiane Danilo ${ }^{5}$ where it was proved that tumors with high levels of HDL cholesterol are the most aggressive.

When we analyze the graphs we realize that there is an endocrine functional elasticity in women that can be measured by the waste of cholesterol, and when we compare this endocrine functional elasticity with the studies that currently exist for the prognosis and treatment of breast cancer such as the estrogen and progesterone receptors 
measured by the immuno- histochemistry methods, as well as the erb2 levels that classify the subgroups of breast cancer, we see that the triple negative tumors ${ }^{6,7}$ and the her- $2+++$ are at their highest percent in the tumors with high cholesterol waste, and our previous studies also show that those tumors with high levels of HDL cholesterol are the most aggressive and that this research suggests us to treat these triple negative tumors and her2, because to use for them a new marker that would be the SRB $1,{ }^{8}$ which is the receptor for HDL cholesterol, this receptor has been investigated in cardiovascular disease and now we see a direct application in breast cancer. In fact, the factors that increase or decrease this receptor have already been described, we can see that vitamin E, corticosteroids and progesterone decrease it, as well as a diet in saturated fats, and in low-waste tumors, where we have to increase the levels of hdl cholesterol, omega 3, unsaturated fats, tamoxifen, sinvastatin, policosanol, etc are described. It is good to emphasize that the highest levels of SRB1 are found in the liver, so the most aggressive tumors with high levels of HDL such as the triple negative ones have metastases in this organ, which would open new possibilities for the development of monoclonal antibodies directed specifically at liver metastases; on the other hand, that explains why drugs such as tamoxifen have no action on liver metastases since it increases HDL cholesterol. On the other hand, breast estrogen receptor positive tumors are mostly among low-waste tumors, there being only a small fraction found in the positive waste close to the equilibrium up to three times increased the normal waste of cholesterol.

This research of course has a great importance in the knowledge of breast cancer in particular and possibly in other types of cancer.

\section{Conclusion}

As we can see in this study there is a correlation between the endocrine functional elasticity (measured by cholesterol waste) and the cellular functional elasticity expressed by the degree of differentiation in breast cancer, allowing us to understand that there is a level of equilibrium expressed in the formula of the waste as zero, there being a functional elasticity within this normal waste that will be published later and that is according to age as it inevitably decreases with the aging of the positive waste to the negative and when it is broken by all the factors that contribute to epigenetic changes ${ }^{9-12}$ such as toxic habits and bad eating habits among others it leads to the appearance of cancer, which is why from the theoretical point of view we believe that the immunological and programmed cell death mechanisms must act in these normal elasticity parameters, since in fact the formula of cholesterol waste is carried out taking into account the normal cholesterol levels reported in the medical literature. We suggest the use of this formula in the radiant treatment in clinical trials since in fact radio curable tumors are differentiated and we already have some therapeutic tips to try to differentiate the tumor and obtain better results by using chemotherapy in the high-waste tumors and factors that increase HDL cholesterol in low-waste tumors it can be done if we know that certain cholesterol metabolites that can promote or suppress breast cancer has been described by Silvente -Poirot and Marc Poirot. ${ }^{13}$ In the other hand the use of the digital sheet in the follow-up of the patients will allow better results in survivor because the changes in the waste of cholesterol allows the Doctor opportune medical intervention.

Because the normal levels of cholesterol and endocrine elasticity are present in the formula of the waste of cholesterol, its use in chemoprevention of breast cancer in the women is feasible. From the point of view of breast cancer pathophysiology ${ }^{14}$ our work suggests that, cancer is produced by a metabolic endocrine imbalance, and this condition could produce changes in cell clones going from dysplasia to oncogenesis, thus supporting the thesis of Dr. MM Karindas about the multicellular origin of cancer. ${ }^{15}$

\section{Acknowledgements}

Journal Cancer Prevention and current Research because its cooperation in the publication of these investigation.

\section{Conflict of interest}

Author declares there are no conflicts in publishing the article.

\section{References}

1. Lazo R, et al. How many times is cholesterol waste increased or decreased at time of breast cancer diagnosis? Its importance in breast cancer treatment (advanced or adjuvant therapy). The Breast. 10(Suppl 1):S19.

2. Roberto Lucio Lazo Carreras, Sonia López Batista, Yoval Aguiar Ferro, et al. The formula of the waste of cholesterol a new way to increase survivor in patients affected from breast cancer. A prospective study. $J$ Cancer Prev Curr Res. 2016;5(3):00159.

3. Lazo R. The four-dimensional thought in cancer treatment. J Cancer Prev Curr Res. 2016;5(4):00171.

4. Vassilios Papadopoulos, Yasaman Aghazadeh, Jinjiang Fan, et al. Translocator protein-mediated pharmacology of cholesterol transport and steroidogenesis. Molecular and Celular Endocrinology. 2015;408(15):90-98.

5. Danilo C. Impact of Cholesterol and Lipoproteins on Breast Cancer. Thomas Jefferson University; 2012

6. Cetin I, Topcul M. Triple Negative Breast Cancer. Asian Pac J Cancer Prev. 2014;15(6):2427-31.

7. Navrátil J, Fabian P, Palácová M, et al. Triple Negative Breast Cancer. Klinicka Onkologie: Casopis Ceske a Slovenske Onkologicke Spolecnosti. 2015;28(6):405-15.

8. Trigatti BL1, Krieger M, Rigotti A. Influence of the HDL Receptor SRB1 on Lipoprotein Metabolism and Atherosclerosis. Arterioscler Thromb Vasc Biol. 2003;23(10):1732-8.

9. Romagnolo DF, Daniels KD, Grunwald JT, et al. Epigenetics of breast cancer: Modifying role of environmental and bioactive food compounds. Mol Nutr Food Res. 2016;60(6):1310-29.

10. Michael Daniel, Tollefsbol TO. Epigenetics of nutrition and environmental effects. Epigenetic linkage of aging, cancer and nutrition. $J$ Exp Biol. 2015;218:59-70.

11. Royston KJ, Tollefsbol TO. The Epigenetic Iimpact of Cruciferous Vegetables on Cancer Prevention. Curr Pharmacol Rep. 1(1):46-51.

12. Carmen Spienza, Jean-Pierre Issa. Diet, Nutrition, and Cancer Epigenetics. Annu Rev Nutr. 36:665-681.

13. Sandrine Silvente-Poirot, Marc Poirot. Cholesterol and Cancer, in the Balance. Science. 2014;343(6178);1445-1446.

14. Erik R Nelson, Ching Yi Chang, Donald P, et al. Cholesterol and Breast Cancer Pathophysiology. Trends in Endocrinology and Metabolism. 2014;25(12):649-655.

15. M Mark Karindas. The multicellular origin of cancer and the clonal evolution of oncogenesis. American Journal of Biomedicine. 2014;2(1):1-14. 